\title{
1 Análisis multicriterio discreto: Un método facilitador para la toma de decisiones en el ordenamiento territorial
}

Degioanni, Américo José"; de Prada, Jorge Dante², Cisneros, José Manuel ${ }^{3}$ y Cantero Gutiérrez, Alberto 4

Servicio de Conservación y Ordenamiento de Tierras. Facultad de Agronomía y Veterinaria. Universidad Nacional de Río Cuarto. Argentina.

Ingeniero Agrónomo (Universidad Nacional de Río Cuarto, 1986) y Doctor en Ciencias Ambientales (Universidad de Alcalá de Henares, España, 1999). Actualmente es Profesor Adjunto por concurso del Departamento de Ecología Agraria, Facultad de Agronomía y Veterinaria, Universidad Nacional de Río Cuarto. Profesor responsable de 2 cursos de grado postgrado en la Maestría de Ciencias Agropecuarias UNRC y en el Doctorado Binacional en Ciencia y Tecnología de la Universidad Federal Rural de Río de Janeiro y UNRC. Ha codirigido y dirigido 13 proyectos de investigación y 2 de extensión. Ha publicado como autor y coautor 3 libros; 14 capítulos de libros; 18 artículos científicos, mas de 80 artículos en eventos científicos y 25 informes técnicos. Participó en 10 eventos científicos nacionales e internacionales. Ha sido presidente del comité organizador del XXV Congreso Argentino de la Ciencia del Suelo 2016. Ha participado en 11 consultorías técnicas para empresas, cooperativas y organismos públicos nacionales, provinciales y municipales. Ha dirigido/codirigido 3 becas de investigación de grado y posgrado, 21 trabajos finales de graduación de la carrera de ingeniería agronómica; 5 tesis de maestría y 1 de doctorado. Ha sido jurado de concursos docentes, tesis de postgrado y de trabajos finales de grado. Ha ejercido funciones de gobierno universitario como consejero directivo titular y como consejero superior suplente de Universidad. Ha sido director y codirector del Dep. de Ecología Agraria. Es consejero titular del Consejo Regional Córdoba de INTA. Ha recibido reconocimientos del Rectorado de la Universidad Nacional de Río Cuarto y de la Honorable Cámara de Diputados de la Nación por trabajos sobre inundaciones.

adegioanni@ayv.unrc.edu.ar

\author{
Américo \\ José \\ Degioanni ${ }^{1}$
}


Ingeniero Agrónomo 1989, Master en Proyectos de Inversión-Universidad Nacional de Córdoba, 1994, M.S. y Ph. D. en Economía Agraria y Recursos Universidad de Connecticut EEUU (2001 y 2005 respectivamente). Investigación en: Ordenamiento y manejo de cuencas, Economía de la conservación de suelo y humedales, Valoración económica de servicios ambientales y Análisis de decisiones multicriterio. Publicaciones: Cuatro libros y diez capítulos de libros, nueve artículos en revista con referato, siete en congresos internacionales; y más treinta en congresos nacionales. Tres premios al mejor trabajo de investigación en la Asociación Argentina de Economistas Agrarios (AAEA), años 2004, 2007 y 2014. Presidente AAEA (2011-12), Director titular (2009-2010), Vocal Titular (2013). Docencia de grado en: Planeamiento Agropecuario, Gestión Ambiental y Proyectos de Inversión agropecuaria. Docencia de Posgrado: Planificación y manejo de tierras (UNRC), Proyecto de inversión agropecuaria y desarrollo (UNRC y Universidad de Connecticut), Gestión Ambiental (UNRC y UFRRJ) Evaluación de Impacto Ambiental (UNRC), y Proyectos de desarrollo, investigación e innovación (UNRC y UNSL). Conferencista en congresos nacionales y encuentros vinculados a la especialidad. Referee de la Revista Argentina de Economía Agraria y de los congresos de la AAEA (2001-2013) y Ciencia e Investigación Agraria-Ed. Pontifica UC de Chile (2010). Extensión y servicios: más de 27 proyectos destinados a productores, entidades intermedias, municipalidad, gobiernos provinciales y gobierno nacional. Gestión institucional: más de 20 proyectos en la Universidad Nacional de Río Cuarto y coordinador de convenios específicos de cooperación: AACREA y UNRC (2005-2013), University of Connecticut y UNRC (2001-2005), Ministerio de la Producción y Empleo de la provincia de Córdoba y UNRC (2005-2006), y Municipalidad de San Basilio y UNRC (2007-2008).

Ingeniero Agrónomo (Universidad de Río Cuarto, 1983), Master Scientiae en Ciencias del Suelo (Universidad de Buenos Aires, 1994) y Doctor en Tecnología Agroambiental (Universidad Politécnica de Madrid, 2010). Actualmente Profesor Titular por concurso del Departamento de Ecología Agraria de la Facultad de Agronomía y Veterinaria, Universidad Nacional de Río Cuarto. Profesor responsable de los cursos "Uso y manejo de Suelos", "Ingeniería en conservación de tierras" y "Manejo de tierras con mal drenaje", en el grado y posgrado. Ha dirigido 7 proyectos y 4 programas de investigación. Ha publicado 25 artículos en revistas científicas, 3 libros y 9 capítulos de libro. Participó en 100 eventos científicos y tecnológicos nacionales e internacionales. Ha dirigido 7 tesis de Magister. Ha sido jurado de tesis de posgrado. $\mathrm{Ha}$ sido miembro de comisiones asesoras de CONICET, CONEAU, FAO y de distintas universidades. Tiene registrada una patente de invención de un dispositivo de labranza. Área de interés: manejo y conservación de suelos; ordenamiento territorial; tecnología de manejo de áreas mal drenadas y sujetas a erosión.

\section{Jorge Dante de Prada²}

José

Manuel Cisneros $^{3}$ 
Ingeniero Agrónomo y Msc en Suelos. Actualmente es Profesor Emérito de la Universidad Nacional de Río Cuarto y Director de la Maestría en Ciencias Agropecuarias de la Facultad de Agronomía y Veterinaria de la misma Universidad. Ha desempeñado cargos como Profesor Titular de la asignatura Uso y Manejo de Suelos. Ha sido Profesor de postgrado en la Universidad de Buenos Aires. Ha sido Rector de la Universidad Nacional de Río Cuarto por tres períodos consecutivos. Ha sido Intendente electo de la Ciudad de Río Cuarto y Diputado de la Nación por un período.

\section{Alberto Cantero Gutiérrez ${ }^{4}$}

\section{Resumen}

El ordenamiento territorial es un proceso social que construye una configuración deseada del territorio. Dicho proceso inicia cuando se acuerda una imagen futura del modelo territorial hacia donde se canalizan los esfuerzos para su concreción. Esta etapa es un problema complejo, pues confluyen diferentes opiniones, intereses y conflictos difíciles de conciliar y, por lo general obstaculizan la ejecución del proceso. El análisis multicriterio es una herramienta que facilita esta primera etapa del OT mediante la selección de la alternativa más favorable desde el punto de vista social, ambiental y económico. Se presenta una aplicación del método para la resolución de un problema de ordenamiento territorial de expansión urbana para la ciudad de Marcos Juárez (República Argentina).

\section{Palabras clave:}

Conflictos sociales - expansión urbana dispersa - decisor político - Estado.

\begin{abstract}
Land Plannig is a social process that builds a desired territory configuration. This process begins when a future image of the territorial model is agreed upon, where the efforts for its concretion are channeled. This stage is a complex problem because different opinions, interests and conflicts are difficult to reconcile, and usually hamper the execution of the process. Multicriteria Analysis is a tool that facilitates this first stage of the LP by select-
\end{abstract}

ing the most favorable alternative from the social, environmental and economic point of view. An application of the method for the resolution of a territorial urban sprawl problem for the city of Marcos Juárez (Argentina) is presented.

\section{Keywords:}

Social conflicts - sprawl urban expansion political decision maker - State 


\section{Introducción}

El ordenamiento territorial es un proceso cuya fase inicial consiste en analizar y proponer la localización correcta de los asentamientos humanos, de la infraestructura y actividades productivas, económicas y sociales, más los servicios básicos de los ecosistemas en el espacio geográfico, considerando los criterios que mejor orientan el interés colectivo de la sociedad, de cara al futuro (Pereyra et al., 2003). Se trata pues, de un proceso social por el carácter de su construcción y porque una vez acordado el modelo territorial deseado su concreción es conducida a través del Estado. En este contexto se reconocen tres etapas:

a) Definir y acordar una imagen objetivo o visión futura de la configuración territorial deseada.

b) Diseñar las estrategias para construir dicha visión. Formulación de programas y proyectos de actuaciones.

c) Ejecutar y gestionar las actuaciones en el territorio. Monitoreo, evaluación y correcciones.

La definición de la imagen objetivo del modelo territorial deseado constituye un problema complejo, dada la diversidad de puntos de vistas, intereses y conflictos en juego. Esto hace prácticamente imposible encontrar una solución que satisfaga los deseos de todos los involucrados que, en caso de no ser resuelto, obstruye la concreción del modelo territorial deseado (de Prada et al, 2014). En este sentido las metodologías de análisis multicriterio constituyen una valiosa herramienta para ayudar al proceso de OT.

El análisis multicriterio es una metodología procedente del campo de la toma de decisiones. Son herramientas para el análisis de alternativas o variables de deci- sión, que son evaluadas a través del cumplimiento de objetivos, y valoradas por criterios cuantitativos o cualitativos. Cuando se trata de seleccionar alternativas finitas y competitivas entre sí, uno de los métodos más utilizados en análisis multicriterio discreto (AMD) es el PROMETHEE I y II -Preference Ranking Organization Method for Enrichment Evaluations (Brans y Mareschal 2005). Este método ha sido utilizado para evaluar y seleccionar propuestas de ordenamiento territorial de expansión urbana por de Prada et al., (2012) y de Prada et al., (2014).

El objetivo de esta comunicación es ilustrar, mediante un ejemplo concreto, cómo se integra el AMD en la resolución de un problema de decisión en el marco del ordenamiento territorial de la ciudad de Marcos Juárez, provincia de Córdoba, República Argentina. Se presenta, en primer término, el problema territorial a estudiar, luego se detalla el procedimiento para la formulación de alternativas y por último se simula la solución seleccionando la alternativa favorable.

\section{Contenidos \\ Caso de estudio: la expansión urbana de la ciudad de Marcos Juárez (Argentina)}

La ciudad de Marcos Juárez (3242' S - $62^{\circ} 6^{\prime}$ O) es una población con aproximadamente 29.000 habitantes, localizada al este de la provincia de Córdoba, República Argentina. Tanto su historia como la vida actual están fuertemente vinculadas a la principal actividad económica de llanura pampeana argentina: la agricultura industrial, extensiva y de exportación. El auge de esta actividad en los últimos decenios ha sido un gran dinamizador del crecimiento urbano por aumento de la población estable y por mayor inversión en la cons- 
trucción de viviendas. La característica de este proceso se inscribe en el modelo de expansión dispersa. Esta forma de ocupación del territorio (poblamiento) involucra una mayor conversión de tierra rural a urbana por la baja densidad del área urbana, incrementa enormemente la distancia de la frontera rural - urbana provocando numerosos conflictos socio-ambientales por incompatibilidad de actividades, encarece y dificulta la cobertura de servicios públicos a toda la población e incrementa el consumo de energía fósil y la emisión de gases de efecto invernadero por un mayor uso del automóvil, entre otros aspectos (McElfish 2007; Ewing et al., 2008; López, 2004). Como consecuencia de esta forma de poblamiento en Marcos Juárez, además de los problemas mencionados, se ha generado un conflicto político institucional entre el Municipio y el Instituto Nacional de Tecnología Agropecuaria (organismo federal cuya misión es el desarrollo de tecnologías para el sector agropecuario) por la competencia en la posesión de un espacio de 152 ha actualmente bajo jurisdicción del organismo federal.

A los efectos de presentar cómo se podría resolver el conflicto emergente y minimizar en el futuro otros conflictos derivados de una expansión urbana dispersa, se propone ensayar el AMD mediante el uso del software Visual PROMETHEE (Mareschal 2013) para formular propuestas de expansión urbana, valorar los criterios y simular la selección de la alternativa más favorable.

\section{Procedimiento para la} formulación de propuestas de expansión urbana. Visión 2040

El procedimiento propuesto diferencia dos fases. La primera fase corresponde al "rol técnico" que es el equipo encargado de identificar intereses, problemas y con- flictos en juego, y de diseñar, junto con los decisores políticos, las propuestas de ordenamiento territorial. Dicho equipo debe considerar los tiempos políticos de los decisores, por lo que debe tener la habilidad de diseñar y parametrizar la propuestas con la información inmediatamente disponible a los efectos de llegar a la solución de compromiso en el menor tiempo posible. Una vez finalizada esta fase se pasa a la segunda, que corresponde al "rol político" del procedimiento. Ello consiste en la valoración y selección de la propuesta más favorable del conjunto, por parte de los decisores encargados de poner en marcha el proceso de OT. La Figura 1 esquematiza el procedimiento de formulación, valoración y selección de alternativas para el caso de expansión urbana, visión 2040 en el marco de AMD.

\section{Diseño de las propuestas de expansión urbana}

Se plantea en primer lugar, como objetivo central, resolver la demanda de espacio rural para urbanizar una ciudad de 35.000 habitantes en 2040, es decir 6.000 habitantes más que los actuales. Por consiguiente, los decisores deberán seleccionar qué espacio se dedicará para alojar la población urbana en el año 2040. Para ello se diseñan 5 propuestas de expansión urbana (PEU1 a PEU5) constituyendo cada una de ellas las alternativas a evaluar y rankear. Cada PEU incluye una zona de amortiguación que es una superficie adyacente al perímetro urbano, que se determina como áreas de protección de la vida urbana (banda de color amarillo en las figuras 2, 3, 4, 5 y 6).

La primera propuesta -PEU1 (Figura 2) constituye la prognosis elaborada sobre la base del diagnóstico, suponiendo que las fuerzas internas y externas de modelación del territorio se mantienen y, por lo tanto, 


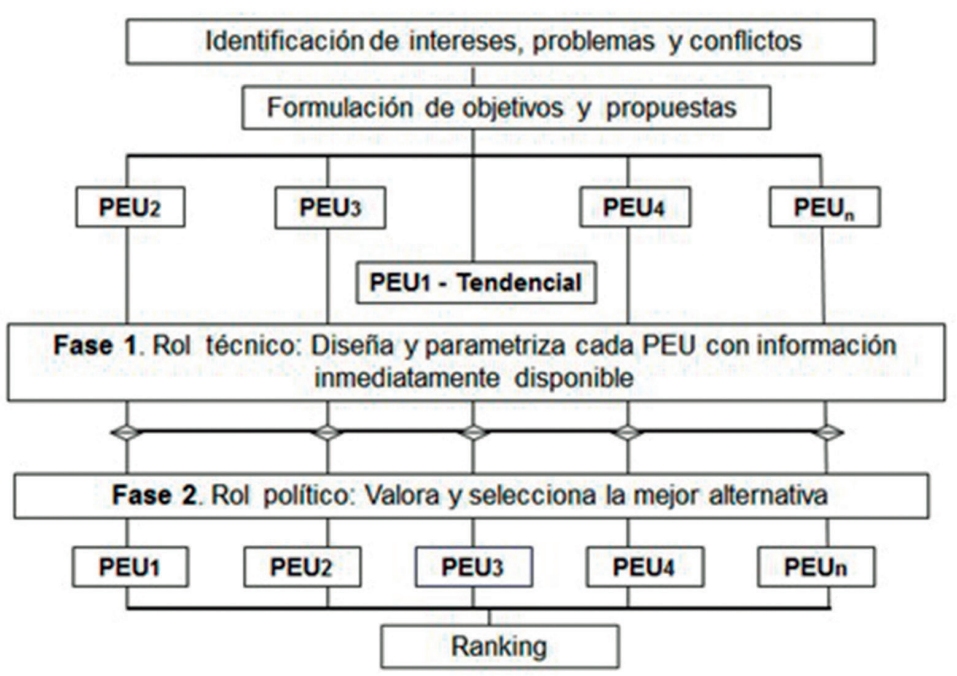

Figura 1: Esquema del proceso de formulación, valoración y selección de alternativas de expansión urbana -visión 2040. (PEU: Propuesta de Expansión Urbana)

se proyectan sin grandes variantes hacia el futuro. Dada la dinámica actual del crecimiento urbano, dicha tendencia será la impuesta por la expansión urbana dispersa. En consecuencia, la PEU1 constituye la situación tendencial manteniendo el actual nivel de intervención en el territorio.

La segunda propuesta de expansión urbana es una alternativa totalmente opuesta a la tendencial: la ecociudad (PEU2 - Figura 3). Este poblamiento se inscribe en el modelo de expansión compacta del crecimiento urbano (Gaffron et al., 2008; Vernay et al., 2010). Dicho modelo promueve una mayor densificación del espacio urbano (entre 45 y 60 habitantes por hectárea) lo que facilita la cobertura de servicios públicos, minimiza el consumo de suelo rural y compacta la ciudad con menor distancia de frontera urbano - rural. Por otro lado, como asigna mayor prioridad al traslado a pie, en bicicleta, transporte público y por último automóvil gasta menos energía fósil y reduce la emisión de gases de efecto invernadero. Propone también disminuir la cantidad de residuos sólidos urbanos a gestionar al promover que los ciudadanos reciclen in situ los residuos orgánicos.

Resulta evidente el contraste entre la PEU1 y PEU2: mayor densificación de la mancha urbana, menor consumo de tierra rural y menor frontera de contacto urbano - rural en la alternativa ecociudad. Las propuestas siguientes son alternativas intermedias entre ambos extremos, y que satisfacen el objetivo de partida: alojar la población estimada para el año 2040 minimizando los actuales y futuros conflictos emergentes de un poblamiento disperso. Para ello se propone consolidar los límites de la mancha urbana actual y destinar un espacio de expansión orientado al noroeste, noreste y sureste de la localidad: PEU3, PEU4 y PEU5 respectivamente (Fig. 4, 5 y 6).

La alternativa PEU5 - Expansión Sureste (Fig. 6) incluye el espacio en conflicto interinstitucional. Es sumamente impor- 


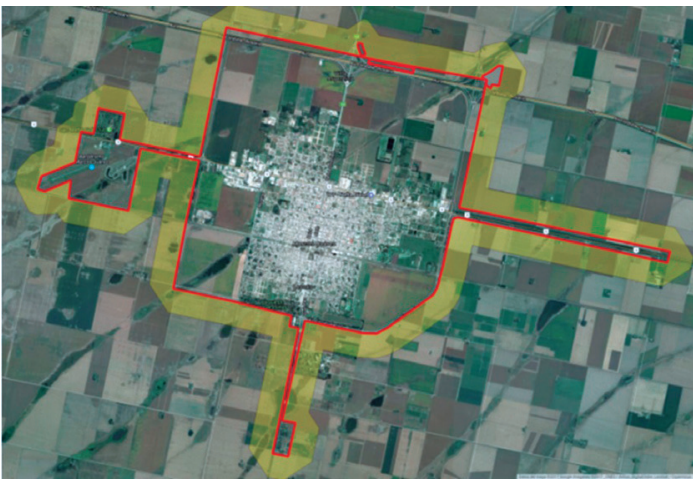

Figura 2: PEU1 - Tendencial

tante no excluir de las alternativas el mismo porque es el punto de partida para poder reunir, en forma conjunta, a los decisores en conflicto para tratar de arribar a un acuerdo en el marco del procedimiento de AMD.

\section{Parametrización de las} propuestas de expansión urbana

Diseñadas las alternativas de expansión urbana -Visión 2040, el paso siguiente es definir los criterios de valoración de cada una de ellas. Un criterio se refiere a un atributo o característica asociada a cada alternativa, y que permite realizar las comparaciones de acuerdo con las preferencias del tomador de decisión. Dichos criterios deben incluir, en la medida de lo posible, las tres dimensiones del desarrollo sostenible: económica, ambiental y social. Para este caso se seleccionaron ocho criterios que se detallan a continuación:

I. Densidad Poblacional: criterio de dimensión social, escala cuantitativa. Mide dos aspectos en simultáneo: a) la cantidad de población a albergar en el territorio y b) el poblamiento. La población futura se proyecta a partir de la tasa de crecimiento demográfico (1\% anual). El poblamiento se estima mediante la densidad urbana: com-

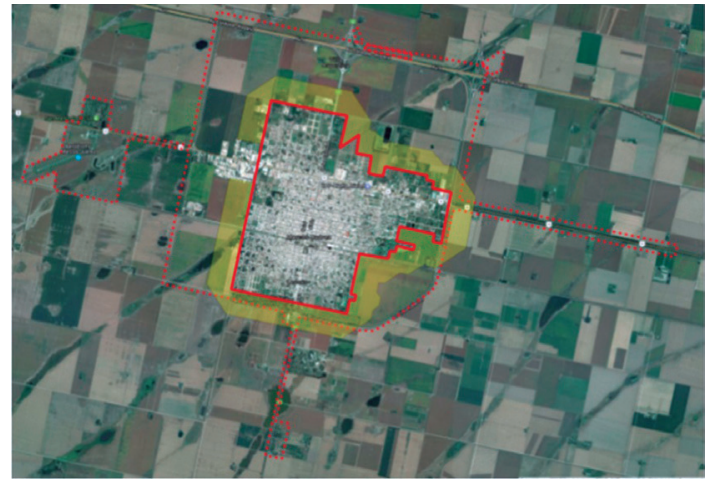

Figura 3: PEU2 - Ecociudad

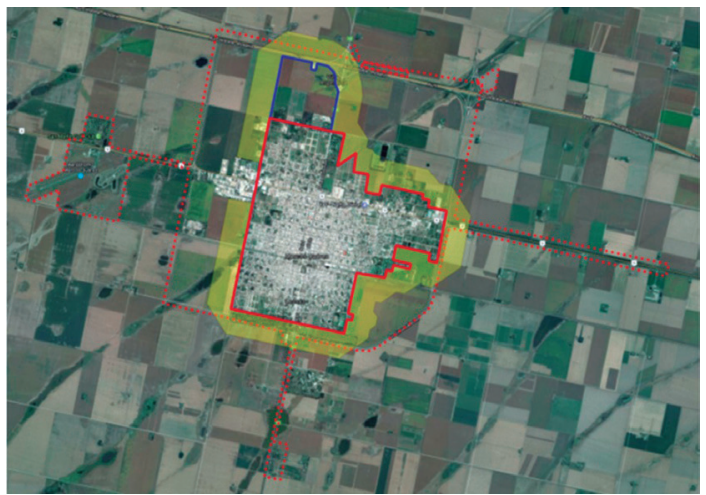

Figura 4: PEU3 - Exp. Noroeste

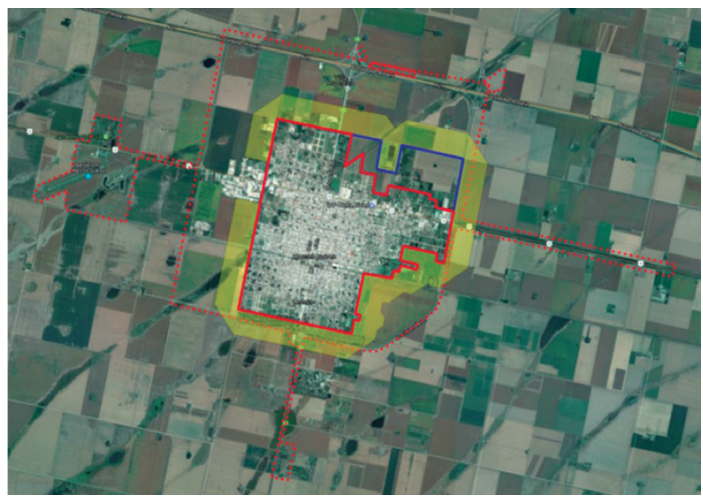

Figura 5: PEU4 - Exp. Noreste 


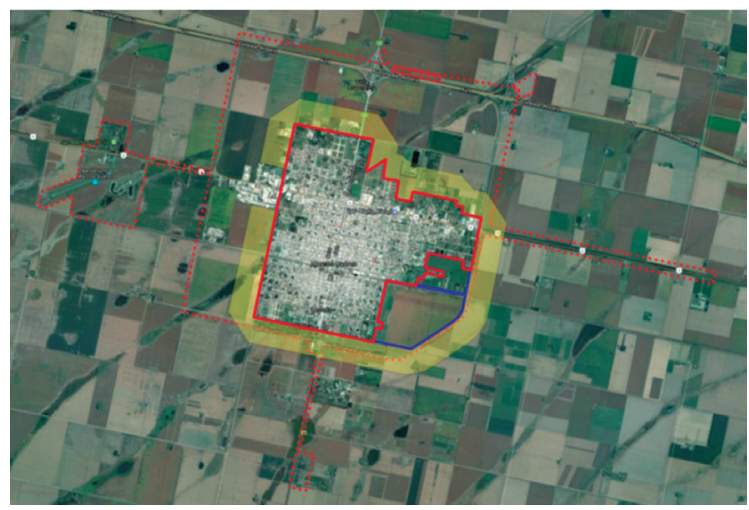

Figura 6: PEU5 - Expansión Sureste

pacto en la PEU 2 - ecociudad: con 45 hab ha $^{-1}$ o disperso en la PEU 1 - tendencial con 10 hab ha-1.

\section{Esfuerzo político institucional:} criterio de dimensión social, escala cualitativa. Mide el esfuerzo que la administración del proceso de OT debe asumir para concretar cualquiera de las alternativas en el territorio (de Prada et al, 2012). La PEU1 tendencial "actuemos como de costumbre, que el territorio se ordena solo" es la de mínimo valor en este criterio. En contraste, la PEU 2 ecociudad es la de mayor valor porque significa cambiar las conductas de los habitantes y del propio Estado para poner en marcha esta alternativa.

III. Conflictividad social: criterio de dimensión social, escala cualitativa. Mide los conflictos socio - ambientales que las alternativas pueden generar al ser instrumentadas en el territorio. Este se mide en función de las distancias de frontera urbana - rural. Además, en este caso en particular, se considera el conflicto interinstitucional (PEU 5).

IV. Riesgo inundación sanitario: criterio de dimensión ambiental, escala cualitativa. Mide el riesgo de inundación y diseminación de enfermedades en el agua subterránea conforme al mapa de profundidad del manto freático o napa freática.

VI. Gestión residuos urbanos: criterio de dimensión ambiental, escala cuantitativa. Mide las toneladas de residuos sólidos urbanos a gestionar anualmente por la administración (recolección, tratamiento, disposición final). Se considera una producción de residuo de 1 kg por habitante y por día. Para el caso de la PEU 2 se asume que hay una reducción del $50 \%$ de los residuos por tratamiento in situ de los mismos.

VI. Extensión redes: criterio de dimensión económica, escala cuantitativa. Mide la longitud de todos los servicios de redes urbanas: calles, electricidad, gas, agua potable, cloacas, fibra óptica, etc. Se calcula mediante operaciones y análisis espacial en SIG.

VII. Pérdida de renta de la tierra: criterio de dimensión económica, escala cuantitativa. Cuando el suelo cambia de uso rural a urbano se pierde la renta económica, puesto que deja de producir bienes agropecuarios. Por tanto, este criterio mide la pérdida en pesos argentinos (\$) que para la sociedad significa cambiar el uso del 
suelo. Se calcula a partir del ingreso bruto de las actividades agropecuarias que se desarrollen en el suelo rural.

VII. Costo de compra de tierra: criterio de dimensión económica, escala cuantitativa. Cuando el suelo pasa de rural a urbano su precio se eleva impulsado por la especulación financiera y establece el valor de mercado de la tierra urbana. Por lo tanto, este criterio mide el valor en pesos argentinos (\$) de comprar la tierra para urbanizar.

Valorado cada criterio es necesario establecer la dirección deseada de cambio del mismo parámetro en AMD, que se denomina objetivo. Cuando se "maximiza" el objetivo a mayor valor del criterio es mejor esa alternativa \{a más mejor\}. Por el contrario, cuando se "minimiza" el objetivo a menor valor del criterio es mejor esa alternativa \{a menos mejor\}. Pe. el criterio Gestión de Residuos Urbanos a menor valor del mismo mejor (menor cantidad de residuo a gestionar anualmente). Por el contrario, si se desea compactar la mancha urbana a mayor valor del criterio densidad urbana, mejor. Cabe consignar que es el tomador de decisión quién establece el objetivo de cada criterio.

\section{Simulación para la selección de la mejor alternativa}

En esta fase del procedimiento AMD el tomador de decisión (TD) es quien establece sus preferencias individuales para evaluar las alternativas. En el método PROMETHEE las preferencias se establecen mediante los objetivos (max o min - Tabla 1) y la ponderación o peso relativo que asigna el TD a cada uno de los criterios. Por ejemplo, un TD puede asignar más peso a criterios ambientales que a criterios económicos, en tanto que otro TD, puede asignar más peso a criterios sociales o económicos y otro puede ponderar en igual magnitud los criterios ambientales y económicos. Normalmente los valores de ponderación se establecen en el rango de 0 y 10. Si un TD asigna valor 0 a la ponderación de un criterio, significa que lo elimina de la evaluación de las alternativas. En tanto, si asigna 10, significa que ese criterio tiene la máxima importancia para él. Valores entre 1 y 9 revelan grados de importancia intermedios. Para el pre-

Tabla 1: Matriz de criterios valorados y objetivo para cada PEU

\begin{tabular}{|c|c|c|c|c|c|c|c|c|}
\hline & $\begin{array}{c}\text { DP } \\
\text { hab.ha-1 }\end{array}$ & $\begin{array}{c}\text { EPI } \\
\text { cualitativo }\end{array}$ & $\begin{array}{c}\text { CS } \\
\text { cualitativo }\end{array}$ & $\begin{array}{c}\text { RIS } \\
\text { cualitativo }\end{array}$ & $\begin{array}{c}\text { GRSU } \\
\text { Tn.año-1 }\end{array}$ & $\begin{array}{l}\text { ER } \\
\text { km }\end{array}$ & $\begin{array}{l}\text { PRT } \\
\text { MM \$ }\end{array}$ & $\begin{array}{l}\text { CCT } \\
\text { MM \$ }\end{array}$ \\
\hline Objetivo & $\max$ & $\min$ & $\min$ & $\min$ & $\min$ & $\min$ & $\min$ & $\min$ \\
\hline PEU 1 & $12^{2}$ & Muy bajo ${ }^{1}$ & Alto & Medio & $14.281^{2}$ & $2.408^{2}$ & $602^{2}$ & $3.430^{2}$ \\
\hline PEU 2 & $37^{1}$ & Muy alto 2 & Medio & Medio & $7.141^{1}$ & $193^{1}$ & $0^{1}$ & $632^{1}$ \\
\hline PEU 3 & 32 & Medio & Bajo ${ }^{1}$ & Bajo $^{1}$ & 10.711 & 371 & 44 & 978 \\
\hline PEU 4 & 31 & Medio & Bajo $^{1}$ & Medio & 9.409 & 399 & 50 & 1.024 \\
\hline PEU 5 & 31 & Alto & Muy alto 2 & Alto $^{2}$ & 9.409 & 434 & 95 & 769 \\
\hline
\end{tabular}

DP: Densidad poblacional, EPI: esfuerzo político institucional, CS: conflictividad social, RIS: riesgo de inundación sanitario, GRSU: gestión de los residuos sólidos urbanos, ER: extensión de redes, PRT: pérdida de renta económica, CCT: costo compra de la tierra; max: maximiza, min: minimiza; superíndice ${ }^{1}$ mejor PEU y superíndice² peor PEU posicionada en función del objetivo y valor del criterio. 
sente ejercicio se relevó la preferencia de 9 TD en una ejercitación del método. En la Tabla 2 se presenta la ponderación promedio y desviación estándar.

El grupo de TD expresa una clara preferencia en los criterios de dimensión ambiental (gestión de residuos sólidos urbanos y riesgo de inundación sanitario) y en uno de la dimensión social (densidad urbana) al asignar los máximos valores de ponderación. Por otra parte no se observa, en general, una dispersión pronunciada en la ponderación a excepción del criterio de dimensión económica Pérdida de Renta de la Tierra. El resultado o ranking de alternativas obtenido con las preferencias del grupo de TD con Visual PROMETHEE se expone en el Gráfico 1.

La alternativa mejor rankeada (valor más próximo a +1) en función de las preferencias de los TD es la PEU3 (Expansión noroeste), seguida de la PEU4 (Expansión noreste) y por último la PEU2 (Ecociudad). Las peores alternativas rankeadas (valores más próximos a -1) son la PEU1 (Tendencial) y PEU5 (Expansión sureste). Estas dos PEU han sido castigadas en su evaluación por los criterios ER, CCT, PRT y DP (PEU1) y los criterios EPI, CS y RIS (PEU5). En cambio, para el resto de alternativas la mayoría de los criterios han favorecido la valoración positiva.

\section{Conclusiones}

Si bien el resultado obtenido es producto de una simulación, se ha podido demostrar que el procedimiento de AMD es un método racional - matemático que permite diseñar, valorar y evaluar alternativas competitivas entre sí, relevando las preferencias individuales o colectivas de los tomadores de decisión en el proceso de ordenamiento territorial. En este caso particular el conflicto interinstitucional que dio origen al problema de decisión, el mismo fue incluido como una alternativa más

Tabla 2: Ponderación promedio y desviación estándar de 9 TD.

\begin{tabular}{|c|c|c|c|c|c|c|c|c|}
\hline Ponderación & DP & EPI & CS & RIS & GRSU & ER & PRT & CCT \\
\hline Promedio & 8,6 & 6,2 & 6,9 & 8,4 & 8,2 & 6,6 & 6,8 & 6,8 \\
\hline Desv. Est. & 1,3 & 1,1 & 1,6 & 1,3 & 1,3 & 1,4 & 2,2 & 1,9 \\
\hline
\end{tabular}

Gráfico 1: Ranking de PEU Visión 2040 para Marcos Juárez.

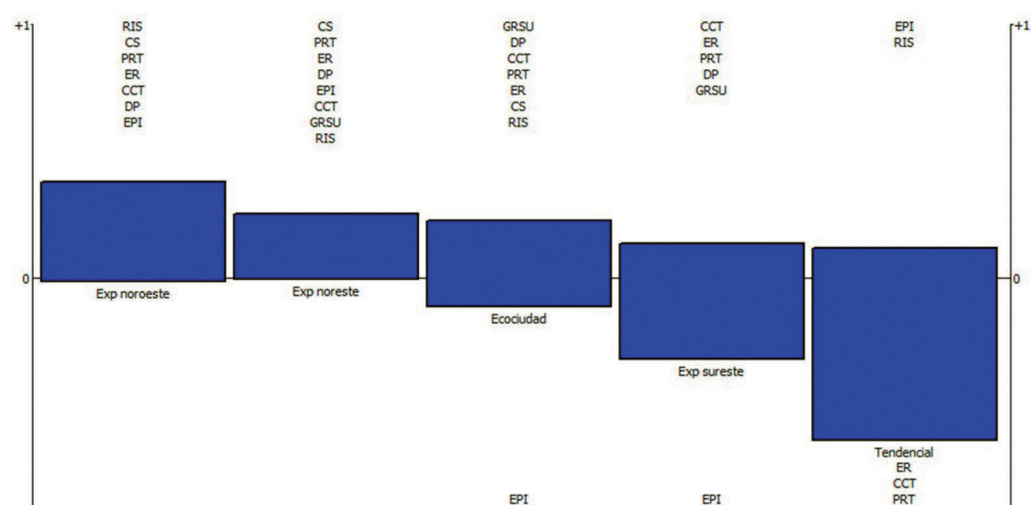

Gráfico 1: Ranking de PEU Visión 2040 para Marcos Juárez 
de expansión urbana. No obstante, bajo los criterios de valoración establecidos y las preferencias simuladas, dicha alternativa ha quedado excluida como opción de expansión urbana.
Es preciso revisar con mayor minuciosidad los criterios establecidos, su valoración y probar las preferencias de decisores del territorio analizado para convalidar el método en la realidad.

\section{Referencias}

Brans, J.P. y Mareschal, B. (2005). Promethee methods. p. 163-195, In Figueira, J., et al., eds. Multiple criteria decision analysis: state of the art surveys, Vol. 78. Kluwer Academic Publishers.

de Prada, J., Degioanni, A., Cisneros, J., Galfioni, M., y Cantero, A. (2012). Diseño y evaluación de propuestas de ordenamiento de territorio: La urbanización sobre tierras rurales. XLIII Reunión Anual de la Asociación Argentina de Economía Agraria. Argentina, Corrientes. (pp. 1-32).

de Prada, J., Degioanni, A., Cisneros, J., Cantero G. A., Gil, H., Tello, D, y Pereyra C. 2014. Preferencias locales para ordenar la expansión urbana: El caso de la ciudad de Río Cuarto, Córdoba, Argentina. Em AAEA. IV Congreso Regional de Economía Agraria y XLV Reunión Anual de la Asociación Argentina de Economía Agraria., CABA, Argentina (pp. 21-23).

Ewing, R., Schmid, T., Killingsworth, R., Zlot, A. y Raudenbush, S. 2008. Relationship Between Urban Sprawl and Physical Activity, Obesity, and Morbidity Urban Ecology. p. 567-582, In MARZLUFF, J. M., et al., eds. Springer USGaffron et al., 2008

Gaffron, P., Huismans, G., y Skala, F., (eds.) 2008. "Proyecto Ecocity. Manual para el diseño de ecociudades en Europa.Libros I y II. La ecociudad: Un lugar mejor para vivir.", pp. 1-137, España.

Lopez, R. 2004. Urban sprawl and risk for being overweight or obese. American Journal of Public Health 94:1574-1579.

Mareschal, B. 2013. Visual PROMETHEE 1.4 Manual.

Mcelfish, J.M. 2007. Ten things wrong with sprawl. Environmental Law Institute, Washington.

Pereyra, C., de Prada, J., Cisneros, J. y Giayetto, O. 2003. Bases Conceptuales y Metodológicas para el Ordenamiento Territorial en el Medio Rural. Cap I. En Bases conceptuales y metodológicas para el ordenamiento territorial en el medio rural. Giayetto O, Plevich O, Lallana V y Pilatti M. Compiladores. 672 Págs. Libroclic Ediciones.

Vernay, A., Salcedo Rahola, T., y Ravesteijn, W. 2010. Growing food, feeding change: Towards a holistic and dynamic approach of eco-city planning. Shenzhen 